\title{
Weak definites and reference to kinds *
}

\author{
Ana Aguilar-Guevara \\ Joost Zwarts \\ Utrecht Institute of Linguistics OTS Utrecht Institute of Linguistics OTS
}

\begin{abstract}
Weak definite NPs do not presuppose the existence of uniquely identifiable entities. In addition, they display a range of other peculiar properties such as sloppy readings in VPs ellipsis, narrow scope interpretations, lexical restrictions and enriched meaning. In order to account for these, in this paper we analyze weak definites as expressions that refer to the same sort of kind individuals that definite generics refer to. We propose that the combination of weak definites with objectlevel predicates is made possible by a lexical rule that lifts object-level relations to 'enriched' kind-level relations.
\end{abstract}

Keywords: weak definites, reference to kinds, definite generics.

\section{Introduction}

One of the traditional ways to characterize the semantics of definite noun phrases is in terms of uniqueness (cf. Russell 1905; Strawson 1950). A definite like the book refers uniquely in a particular context in the sense that it presupposes that there is exactly one entity in that context satisfying its description. Several authors have pointed out that some uses of definites do not display this property (Löbner 1985; Ojeda 1993; Birner \& Ward 1994; Poesio 1994; Abbott 2000; Barker 2005; Carlson \& Sussman 2005; Epstein 2002). We will refer to these problematic definites as weak definites, but the particular subclass that has been described in Carlson \& Sussman 2005 will be our primary focus. The following example illustrates this class.

(1) Lola is reading the newspaper.

As the newspaper does not seem to refer to a unique newspaper, sentence (1) is perfectly suitable to describe, for example, a situation in which Lola is sitting on the

* This research is supported by the Netherlands Organization for Scientific Research (NWO). We would like to thank Seth Cable, Chuck Clifton, Bart Geurts, Jesse Harris, Martin Everaert, Lyn Fraizer, Ellen-Petra Kester, Bert Le Bruyn, Louise McNally, Barbara Partee, Floris Roelofsen, Tom Roeper, Henriëtte de Swart, Jolien Scholten, Florian Schwarz, the participants of the Semantics group UiL OTS, and the audience of SALT 20 for very helpful discussions. Of course, the usual disclaimers apply. 
sofa of a waiting room reading a newspaper, and there are some more newspapers lying next to her.

We will argue in this paper that weak definites do refer to uniquely identifiable entities, but these are abstract objects rather than concrete ones. More specifically, we will claim that weak definites are like singular definite generics in having kindlevel reference. In this way, the newspaper in the example above does not refer to any newspaper in particular, but to the general type or kind that corresponds to the noun newspaper. Analyzing weak definites in this way not only allows us to maintain the ordinary uniqueness semantics of the definite article, but it also explains various mysterious aspects of the behavior of weak definites.

This paper is structured as follows. In section 2, we first describe the behavior of weak definites. In section 3, we will consider, and reject, a possible approach in terms of minimal situations. Our own kind-based analysis is worked out in section 4 . After that we close the paper with some discussion of both the potentials and the limitations of our approach.

\section{Weak definites}

To characterize weak definites, we will build on some observations mostly made by Carlson \& Sussman (2005). The analysis we propose in section 4 aims to account for all of them.

To diagnose the non-unique reference of weak definites, Carlson and Sussman use the possibility of sloppy identity in elliptical contexts. Contrast the following examples.

(2) a. Lola went to the hotel and Alice did too.

b. Lola went to the hospital and Alice did too.

The interpretation of sentence (2a), which contains a definite that is read as regular in English, presupposes that Lola and Alice went to one and the same hotel. In contrast, the hospital in (2b) receives a weak reading and therefore the sentence does not imply that Lola and Alice necessarily went to the same hospital. Notice that this definite can also be read as regular (i.e., as referring to a specific hospital), in which case the sloppy reading effect is blocked.

The non-unique reference of weak definites is also seen when they interact with quantified expressions. In an example like (3a), the hospital seems to take narrow scope and therefore to allow a distributive reading. Again, such an effect is impossible for a regular definite like the hotel, as illustrated in (3b).

a. Every boxer was sent to the hospital. 
Weak definites and reference to kinds

\section{b. Every boxer was sent to ${ }^{\#}$ the hotel. ${ }^{1}$}

In addition to this primary non-uniqueness property, weak definites display a range of other peculiarities. For example, weak definites only occur as objects of certain verbs and prepositions, which vary from case to case:

a. Sally checked the calendar. vs. Sally read ${ }^{\#}$ the calendar.

b. Lola went to the store. vs. Lola went around ${ }^{\#}$ the store.

Likewise, not just any noun can form the head of a weak definite:

a. You should see the doctor. vs. You should see ${ }^{\#}$ the surgeon.

b. Lola listened to the radio. vs. Lola listened to ${ }^{\#}$ the tape recorder.

Similarly, the number morphology of the head of a weak definite, which can be plural or singular, cannot be altered without losing the weak reading:

a. Sally checked the calendar. vs. Sally checked ${ }^{\#}$ the calendars.

b. Lola went to the mountains. vs. Lola went to ${ }^{\#}$ the mountain.

Another, closely related restriction involves modification. In general, weak readings disappear if the nouns are modified. Only adjectives that establish subtypes of the nouns they modify are acceptable:

(7) a. Lola is in \#the new hospital. vs. Lola is in the medical hospital.

b. You should see ${ }^{\#}$ the doctor who works in the medical center. vs. You should see the eye doctor

Weak definites typically occur in object position. When the same definites are the subjects of episodic sentences, they can only be interpreted as referring to specific entities, as illustrated in (8). However, when these definites occur as the subjects of generic sentences, like in (9), they can receive interpretations that seem quite close to weak definite interpretations.

a. $\quad$ The newspaper disappeared.

b. $\quad$ \#The hospital closed at five o' clock.

(9) a. The newspaper brings people their daily news.

b. The hospital is the place where most children are born.

Sentences with weak definites usually carry more information than what seems to be conveyed by the straightforward composition of its constituents. This meaning

1 The \# symbol indicates that a weak reading is not possible for the definite in question. The reader is invited to verify that with the sloppy identity test. 
enrichment is stereotypical in the sense that it invokes the most common circumstances under which the event referred to by the sentence could happen (e.g. the most common circumstances under which one goes to the store is to do shopping). Crucially, the stereotypical meaning of a sentence with weak definites entails the non-stereotypical one:

$$
\begin{aligned}
& \text { a. Eva called the doctor = Eva called the doctor + Eva asked for medical } \\
& \text { assistance. } \\
& \text { b. Lola went to the store = Lola went to the store + Lola did shopping. }
\end{aligned}
$$

Interestingly, if the extra meaning effect is blocked, then the weak reading of the definite also tends to disappear:

a. Eva called ${ }^{\#}$ the doctor to talk about fund raising and budgeting for churches.

b. Lola went to ${ }^{\#}$ the store to pick up a friend.

The last property of weak definites we mention here is their limited capacity to establish discourse referents, as illustrated in (12). As some recent experimental findings suggest for Dutch (Scholten \& Aguilar-Guevara 2010), it seems that these definites are not easy antecedents for anaphors in later discourse, although this still has to be tested in a thorough way.

a. Alice did a solo on the saxophone. ?She did not realize it was out of tune.

b. Lola listened to the radio until she fell asleep. ?She turned it off when she woke up in the middle of the night.

\section{Towards an account}

When confronted with the phenomenon of weak definites, there are a couple of theoretical choices one needs to make. First, one must decide whether or not to treat VPs that embed weak definites (e.g. take the bus and go to the doctor) as expressions with a compositional semantics. Following Nunberg, Sag \& Wasow (1994), we recognize their idiomatic nature as they involve stereotypical meanings and distributional restrictions. However, we consider them idiomatically combining expressions rather than mere idiomatic phrases, according to the nomenclature of these authors, mainly because their meaning seem to be compositionally determined by their parts. For example, the meaning contributions of the verb go, the preposition to, and the noun phrase the doctor in go to the doctor, are still evident independently of the stereotypical enrichment added on top of the normal interpretation of the construction (i.e., to ask for medical assistance). 
Weak definites and reference to kinds

Second, one must decide to privilege or not the traditional uniqueness analysis of the definite article. Our position with respect to this issue is determined by the normal semantic conservativity: we assume that the definite article in weak definites has the same meaning that it has in ordinary contexts, mapping a singleton set to its unique member.

These two theoretical preferences motivate the main goal of this paper, namely, to develop a compositional semantics of weak definites while maintaining the uniqueness presupposition of the definite article. To pursue this, there are two possible strategies. We will call them the minimal situation strategy and the abstract referent strategy respectively:

A Minimal situation strategy: Weak definites refer to ordinary objects that are unique in sufficiently restricted minimal situations.

B Abstract referent strategy: Weak definites refer to abstract objects that have a context-independent uniqueness.

The minimal situation strategy has been prefigured in Löbner 1985 and it is also naturally suggested by the general situational uniqueness approach to the definite article developed by Schwarz (2009). Very roughly speaking, a definite noun phrase picks out its uniquely identifiable referent from a minimal situation $s$. If uniqueness is not satisfied in that situation $s$, then it is assumed that there is a situation $s^{\prime}$ part of $s$ which contains the unique referent. Applied to our example (1) this means that we simply have to 'zoom in' enough to find the minimal situation in which there is one single newspaper that Lola is reading.

There is an empirical problem with this approach. Consider the following example:

Lola took the train from A to B.

Suppose that Lola traveled by train from A to B, but she actually made a transfer halfway. Then she actually took two trains. The whole sentence describes a situation with two trains and it is absolutely unclear how we pick out a smaller situation in which there is one train.

Our conclusion then is that the minimal situation strategy runs into a crucial problem: the potential of weak definites to refer to more than one object in the minimal situation described by the sentence. Furthermore, it is unclear how this approach can account for the other peculiar properties of weak definites.

We therefore assume that a weak definite refers to unique entities but at a more abstract level than ordinary objects. More specifically, we will propose in the following section that weak definites refer to kinds, which can have single or 
multiple instantiations in a situation. In (13), the weak definite refers to one unique abstract train that is realized by two specific trains in the situation described by this sentence. In this way, by distinguishing two levels, we can account for the uniqueness (satisfied at the higher, abstract level) and the plurality (at the lower, concrete level).

\section{Reference to kinds}

The analysis for weak definites that we develop in this section has four major steps. First, we treat weak definites as referring to kind individuals which are of the same sort that definite generics refer to (section 4.1). Second, we will work out how these kinds are implicitly instantiated in the semantics of a sentence (section 4.2). Third, we will address the stereotypical enrichments accompanying weak definites. Fourth, we argue that weak definites are possible in sentences because a lexical rule lifts object-level verbs to kind-level verbs with additional usage restrictions (section 4.4).

\subsection{The nature of the kind term}

There is another class of uses of definites that seems to present a problem for a uniqueness approach as they occur in sentences that do not predicate about one and only one individual, namely definite generics. (14) gives two examples from Krifka 1995. Sentence (14a) is about individual lions in general, whereas (14b) is about the species of the lion (Panthera leo) as a whole. In neither of the two cases does the lion refer to one uniquely given individual lion.

a. The lion roars when it smells food.

b. The lion vanished from Asia.

Dayal (2004) and Krifka (2003) assume that these two definites refer to uniquely identifiable (typically well-established) kind individuals. This kind reference is derived by compositionally combining the standard iota meaning of the definite determiner with a taxonomic common noun, that is, a noun that instead of having its denotation in the domain of ordinary objects, has its denotation in the domain of kinds and sub-kinds (i.e., denotes properties of (sub)kinds, see also McNally \& Boleda 2004 for arguments in this direction). Accordingly, the noun lion in (14) denotes a singleton set containing a unique kind in a taxonomic domain.

The use of definite generics falls out naturally in a context in which the taxonomic domain is restricted to a particular taxonomic level. For instance, if the universe of discourse is restricted to general species (like whale, lion, dog, tiger) and does not include types of lions (like the African lion, the Asiatic lion, etc.), then there is one unique lion kind (namely the Panthera leo), the denotation of the taxonomic noun 
lion is the singleton set $\{$ Panthera leo $\}$ and the definite the lion denotes its single element, namely Panthera leo. In other words, the lion translates as $l x_{k} \cdot \operatorname{lion}_{T}\left(x_{k}\right)$, where the subscript $\mathrm{T}$ indicates that the predicate lion is taxonomic and the subscript $k$ that the variable ranges over kinds of lions. In this way, kind reference with definites can be explained without specific assumptions.

It is important to contrast the direct kind-reference of definite generics with the Carlsonian kind-reference of bare plurals, which is derived by applying the 'down' operator $\cap$ to a plural property to get the corresponding maximal entity that satisfies that property (see Dayal 2004, Chierchia 1998, and Krifka 2003 for discussion). This distinction has several consequences. First, whereas the possibility of mapping a property by ${ }^{\cap}$ to a corresponding plural kind can occur in almost every context, singular definite generics need to be supported by a taxonomic domain from which they obtain their denotation. That explains why only reference to so-called well-established kinds seems felicitous (cf. Carlson 1977; Krifka 1995) ${ }^{2}$

(15) a. The coke bottle is easy to recycle. vs. ?The green bottle is easy to recycle.

b. Coke bottles are easy to recycle vs. Green bottles are easy to recycle.

Second, unlike plural kinds, the atomic kind individuals denoted by definite generics do not allow predications about sums (16a), dependent plurals (16b), or reference to mass nouns (16c). This is because the atomic kind that the definite generic refers to is incompatible with the cumulativity that is inherent to mass nouns and plural predication.

a. *The tiger is numerous.

b. The dodo had purple beaks $\neq=$ 'each dodo had only one beak'.

c. *The rice is produced locally.

Taking this kind-referential nature of generic definites as a point of departure, let us now return to weak definites. Our proposal is that these expressions, just like generic definites, refer to atomic kind individuals. For example, the definite the newspaper in the episodic sentence (1) as well as in the characterizing sentence (9b) (repeated in (17a) and in (17b) respectively) refers to the unique kind of which all newspapers (and only newspapers) are individual instantiations, which can be represented by the term $\imath x_{k}$.news paper $\left(x_{k}\right)$. This term then denotes the 'generic' newspaper.

2 As Dayal (2004) points out, the context might help to 'establish' a kind, which shows that the 'well-establishedness' is not an absolute ontological notion, but a property that depends on specific and variable cultural factors. 
a. Lola is reading the newspaper.

b. The hospital is the place where most children are born.

Analyzing weak definites as kind referring expressions helps us to explain some of the properties we mentioned above. First of all, it explains the presence of a definite article in these phrases despite the lack of the uniqueness presupposition at the level of ordinary entities. It is the uniqueness of the kind that licenses the definite article, just like in definite generics in the approach of Dayal (2004).

Second, we can account for why modification with weak definites is so restricted. The noun in a weak definite has a taxonomic interpretation (a set of kinds) and it can only be modified by expressions that operate on that kind denotation, i.e., that are taxonomic themselves in a sense. The adjective medical is such a modifier that takes the set of 'hospital kinds' and maps it to the singleton set of the medical hospital. The adjective new, however, typically operates at the level of ordinary objects and is therefore incompatible with taxonomic nouns, which is why the new hospital cannot be a weak definite. This account of modification in weak definites fits in nicely with the kind-based account of relational nouns in McNally \& Boleda 2004.

There is an apparent problem with the idea of weak definites referring to kind individuals, namely that in contrast to characterizing sentences like (17b), episodic sentences like (17a) are not directly about kinds, but rather about instantiations of those kinds. That brings us to the next step of our analysis.

\subsection{The kind term and its realization}

Every semantic analysis that uses kinds also needs to consider how these kinds are instantiated or realized by particular individuals. Following Carlson (1977) we assume a realization relation $R$ between individual objects and the kinds that they are realizations of. If $\mathrm{N}$ is the newspaper kind, then $R\left(x_{i}, N\right)$ means that the individual $x_{i}$ is a realization of that kind, i.e., a specific newspaper. Crucially, such a realization can be an atomic entity but also a sum of entities. For example, if $\mathrm{T}$ is the kind of object that corresponds to the noun phrase the train, then not only is every individual train a realization of $\mathrm{T}$, but also every plural sum of trains. This makes it possible for sentence (13), repeated in (18), to refer to a situation in which Lola actually took two trains to get from A to B. Even though there is a plurality of trains here at the lower level of realizations, there is still uniqueness at the higher level of the kind.

Lola took the train from A to B.

Interestingly, there are also cases of plural weak definites that seem to challenge our idea of singular kinds: 
Weak definites and reference to kinds
a. Alice went to the mountains.
b. John went to the movies.
c. Lola is doing the dishes.

Treating these definites as referring to singular, atomic kinds, seems at first sight to contradict the plurality of the nouns they contain, which requires reference to sums of entities. However, we believe that these examples also fit into our theory once we recognize that the nouns are a sort of pluralia tantum or collective nouns and are not really semantically plural. For example, the plural noun dishes does not compositionally relate to a singular noun dish, as the set of sums built up out of individual dishes, but it refers to an individual collection of dirty dishes.

Let us make more precise now how kinds and realizations are represented in the logical form of a sentence with a weak definite using again the example of Lola reading the newspaper. In (20a), Lola is involved in a reading situation with one or more instantiations of the newspaper kind. Analyzing this in Neo-Davidsonian terms (cf. Parsons 1990), (20a) corresponds to a reading event of which Lola is the agent, and instantiations of the newspaper kind $\mathrm{N}$ are the theme, as represented in (20b).

$$
\begin{aligned}
& \text { a. } \quad \text { Lola is reading the newspaper. } \\
& \text { b. } \exists e[\operatorname{read}(e) \wedge A g(e)=\text { lola } \wedge R(T h(e), N)] \\
& \text { c. } \quad \exists e \exists x_{i}\left[\operatorname{read}(e) \wedge A g(e)=\operatorname{lola} \wedge T h(e)=x_{i} \wedge R\left(x_{i}, N\right)\right]
\end{aligned}
$$

The verb is a one-place predicate over events and thematic roles are functions from events to participants (atoms or sums). Note that in (20b) there is no existential quantifier over realizations of the newspaper, as in the alternative representation (20c). Following Espinal \& McNally (to appear) we use the functional expression $T h(e)$ directly as the first argument of the realization relation, thereby avoiding the existential quantifier. Espinal \& McNally (to appear) use such a representation to account for the deficient referential status of bare singular nouns in Spanish. We use it here to account for the intuition that weak definites do not introduce discourse referents at the individual level, even though they refer to kinds. Given appropriate assumptions about the way discourse reference is established in a logical form through existential quantifiers, the representation (20b) predicts that weak definites do not allow anaphora, as we discussed in section 2 (example (12)). This implies that even though truth-conditionally equivalent, the representations (20b) and (20c) have different discourse properties.

With a representation like in (20b), with individual realizations of the kind being tied to the event variable in a local way, we can account for the presence of sloppy readings in VP ellipsis constructions as well as narrow scope reading in sentences with quantified expressions. Sloppy identity in VP ellipsis occurs because the Th function is specific for each of the two events expressed in a sentences like (2), 
repeated in (21a), and because each sentence has its own existential event quantifier.

a. Lola went to the hospital and Alice too.

b. $\exists e[$ go-to $(e) \wedge R(T h(e), H) \wedge A g(e)=$ lola $] \wedge \exists e[$ go-to $(e) \wedge R(T h(e), H) \wedge$ $A g(e)=$ alice $]$

Similarly, the narrow scope effect is due to the event quantifier having narrow scope with respect to the scope bearing operator and the thematic role being dependent upon the event variable:

a. Every boxer went to the hospital

b. $\quad \forall y[\operatorname{boxer}(y) \rightarrow \exists e[$ go-to $(e) \wedge R(T h(e), H) \wedge A g(e)=y]]$

Having the realization relation at our disposal we can also be a bit more specific about the generic use of weak definites, shown in (9). The characterizing statement that is made about a kind involves generic quantification over realizations of that kind, as it is typically the case for a derived kind predication (Krifka 1995). A partial semantic representation for the sentence in (23a) is given in (23b), with GEN being a generic generalized quantifier that takes the set of realizations of the newspaper kind as its restrictive term and the set of things that bring people their daily news as the nuclear scope.

a. The newspaper brings people their daily news.

b. $\operatorname{GEN}\left(\lambda x_{i}\left[R\left(x_{i}, N\right)\right]\right)\left(\lambda x_{i}\left[x_{i}\right.\right.$ brings-people-their-daily-news $\left.]\right)$

\subsection{Stereotypical usage patterns}

Stereotypical enrichment shows up with many (and maybe all) weak definites. We take enrichment for an example like (24a) to mean that there is an additional restriction on the event being quantified over, in this case the events in which Alice does not just go to a building that we would classify as a hospital, but furthermore, that she was involved in the stereotypical function of hospitals (being a patient, nurse, or doctor). For simplicity, we will use a two-place predicate $U(e, K)$ to indicate that $e$ is a stereotypical usage of a kind $K$ and assume that this usage information is important for every weak definite. Notice that we take stereotypicality to be a property at the level of kinds and not at the level of instantiations. In (24), through the relation $\mathrm{U}$ the hospital kind $\mathrm{H}$ is associated to a set of events in which hospital instantiations function in ways that are stereotypical for $\mathrm{H}$.

a. Alice went to the hospital.

b. $\exists e[$ go-to $(e) \wedge A g(e)=$ alice $\wedge R(R e f(e), H) \wedge U(e, H)]$ 
What (24b) denotes is the set of events in which Lola is approaching and the Ref(erence object) of this change of position is a realization of the hospital kind that fulfills in that event its stereotypical function. ${ }^{3}$

Notice that we are treating this extra meaning invoked by sentences with weak definites simply as part of their semantics. This seems adequate, given that, as we can see in (11) and (25), canceling the enrichment does not seem to be possible while maintaining the weak interpretation of the definite. ${ }^{4}$

a. John called ${ }^{\#}$ the doctor, but not for medical reasons.

b. Lola checked ${ }^{\#}$ the calendar, but not to find out about dates or appointments.

We believe that the usage patterns of weak definites are also crucial for understanding why there are lexical restrictions on weak definites, although we can only scratch the surface here, to be honest, both empirically and theoretically. First of all, stereotypical usage patterns are not available for any arbitrary noun, but are restricted to those nouns that somehow can support stable, specific, and stereotypical patterns of use in a particular language community.

Some nouns are too general to support such patterns (like building). The set $\lambda e[U(e, B)]$ of stereotypical usage events for the building kind $B$ is empty because it is impossible to come up with activities that are typical for buildings, given that there are so many different types of buildings, each with their own functions and uses. Other nouns are specific, but they still have not associated to them a non-empty set of typical usage events. This is the case for tape recorder, for instance. In our proposal the set $\lambda e[U(e, T)]$, i.e., the set of usage events of the tape recorder kind $T$, is empty, because the tape recorder does not have a very clear, stereotypical activity with it. The noun hotel does not form weak definites, even though it seems to have a clear function. This shows that there must be additional constraints on the mapping from kinds to stereotypical usage events that are still unclear at this point.

On the other hand, even if a noun can form a weak definite, it does still not freely combine with all verbs and prepositions. In order to understand this restrictiveness we have to look a bit closer at the combinations that we actually find. Some nouns (e.g. store, hospital, beach, park) have a weak reading with verbs and prepositions that all entail locations (e.g. go to, be at, visit, leave). Another class of nouns, that

3 We have simplified things here by assuming just one central event. However, the hospital does not fulfill its stereotypical function in the going event $e$ itself, but in the event $e^{\prime}$ that is the end state of the going. We have to leave that important distinction here. Also we have taken for granted that the 'user' of the hospital in $U(e, H)$ is the same person as the theme of the event $e$.

4 It is imperative to assess in future work the precise semantic-pragmatic status of this semantic enrichment. As Stvan (1998) has suggested for some bare singular nominals, this kind of enrichment is a close kin of conversational implicatures (cf. Horn 1984; Levinson 2000). 


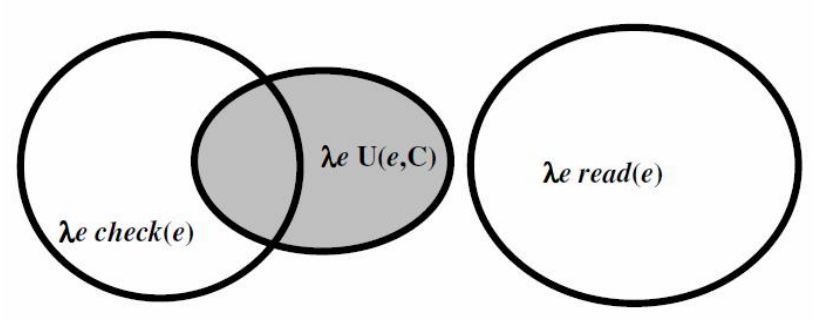

Figure 1 Checking the calendar vs. Reading the calendar

refer to artifacts with very specific patterns of use, select verbs that either refer to that use directly or that express an essential condition for it (read-newspaper, check - calendar, listen - radio, play - piano, open - window). The noun doctor for instance combines with both locative predicates (go to, visit) and with more specific verbs (consult, call). As one can see, all these verbs and prepositions are somehow closely associated to the usage patterns of the kind that correspond to a weak definite. More precisely, the verb either directly expresses a typical activity for the noun (e.g. play - piano, or it expresses a necessary condition (e.g. go to - store, where going to a store is a necessary condition for using that store to buys things.

Formally speaking, all this means that in order for a verb + weak definite combination to be possible, the set of events corresponding to those verbs should overlap with the set of events representing the stereotypical usage of the kind denoted by the weak definite. Suppose that $U$ assigns a non-empty set of usage events to a kind $\mathrm{K}$, then this set might be too restricted to allow overlap with the set of events defined by the verb. The kind $\mathrm{C}$ corresponding to the noun calendar, for instance, has a set $\lambda e[U(e, C)]$ of events that are stereotypical usages of the calendar. But, reading is not a typical activity of calendars, while checking is. In other words, there is an empty intersection of $\lambda e[U(e, C)]$ with the set $\lambda e[\operatorname{read}(e)]$ and therefore (4a), repeated in (26a) with (26b) as its representation, is always false. The intersection of $\lambda e[U(e, C)]$ with the set of checking events is not empty. This is also graphically illustrated in Figure 1.

a. Sally read ${ }^{\#}$ the calendar.

b. $\exists e[\operatorname{read}(e) \wedge R(T h(e), C) \wedge U(e, C)]$

In the same way, we can explain (4b), repeated in (27a). The store has a non-empty set of usage events, but these all require that the agent of the event is located at the store. Lola cannot do shopping in a store if she is not there at some point. But, this is exactly what the preposition around excludes. Given the way the world is, (27b) also expresses a contradiction, because the set of going around the store events does 
Weak definites and reference to kinds

not overlap with the set of events in which the store is used in its typical function.

a. Lola went around ${ }^{\#}$ the store.

b. $\exists e[\operatorname{go}-\operatorname{around}(e) \wedge A g(e)=\operatorname{lola} \wedge R(\operatorname{Ref}(e), H) \wedge U(e, S)]$

\subsection{Combination of object-level verbs and weak definites}

Let us now turn to the compositional structure of sentences with weak definites. If we assume, with Kratzer (1996), that external arguments are introduced syntactically and are not part of the lexical semantics of verbs and that the existential quantifier over events is introduced by the tense-aspect system, then we can represent VP meanings as follows:

a. read the newspaper.

b. $\lambda e[\operatorname{read}(e) \wedge R(T h(e), N) \wedge U(e, N)]$

a. go to the hospital.

b. $\lambda e[$ go-to $(e) \wedge R(\operatorname{Ref}(e), H) \wedge U(e, H)]$

To obtain the verb meanings, we abstract over the kind argument:

a. $\quad$ read: $\lambda x_{k} \lambda e\left[\operatorname{read}(e) \wedge R\left(T h(e), x_{k}\right) \wedge U\left(e, x_{k}\right)\right]$

b. $\quad$ go to: $\lambda x_{k} \lambda e\left[\right.$ go-to $\left.(e) \wedge R\left(\operatorname{Ref}(e), x_{k}\right) \wedge U\left(e, x_{k}\right)\right]$

What we have in (30) are special kind-level meanings for the verbs read and go to. These meanings can only apply to kinds to give us enriched meanings. This creates a systematic ambiguity between the ordinary object-level meaning of verbs and their special kind-level meaning. We propose a lexical rule that shifts verbs or prepositions from an object-level meaning to a kind-level meaning:

(31) Kind Lifting Rule: If $V$ is a transitive verb (or verb-preposition combination) with interpretation $\left.\lambda x_{i} \lambda e\left[V(e) \wedge T h(e)=x_{i}\right)\right]$ then $V$ also has the meaning $\lambda x_{k} \lambda e\left[V(e) \wedge R\left(T h(e), x_{k}\right) \wedge U\left(e, x_{k}\right)\right]$.

This lexical rule can be seen as a general type-shift function (or rather, sort-shift function). It can in principle apply to any verb, but, as we already saw, its applicability is restricted through the usage patterns of the kind that it applies to. For example, take the interpretation of call to be $\left.\lambda x_{i} \lambda e\left[\operatorname{call}(e) \wedge T h(e)=x_{i}\right)\right]$, that is, a function from individuals to events in which those individuals are called. On the basis of the rule in (31), we can also interpret call as $\lambda x_{k} \lambda e\left[\operatorname{call}(e) \wedge R\left(T h(e), x_{k}\right) \wedge U\left(e, x_{k}\right)\right]$. In this way, the relation between persons and events in which those persons are called is shifted to a relation between kinds and events in which realizations of that kind are called as part of their stereotypical function. This function can then be applied to the 
Ana Aguilar-Guevara and Joost Zwarts

denotation of the doctor (which is the kind D) so that we get the following meaning:

$$
\lambda e[\operatorname{call}(e) \wedge R(T h(e), D) \wedge U(e, D)]
$$

(32) corresponds to the set of events in which instantiations of the doctor kind are called with a stereotypical purpose, namely to ask for medical assistance. In the case of call, the doctor kind might be the only kind for which the kind-level function is defined, but there are other verbs, like visit, that have a wider domain of kinds to apply to (hospital, store, and probably other institutions).

We have presented the Kind Lifting Rule as a general rule, applying to any verb, and yielding a function that can apply to any kind. The restrictions on which verbs combine with which nouns are derived from the usage events that are assigned to a kind. Many verbs never combine with a weak definite, simply because their set of events does not overlap with the usage events of any kind. Other verbs (like read), only combine with the usage events of certain kinds (e.g. newspaper), but not of other kinds (e.g. calendar).

\section{Conclusion}

We have proposed an analysis of weak definites that departs from the assumption that these are expressions that, although idiomatic, have a compositional meaning. We treat these as referring to kind individuals that are instantiated when they combine with object-level predicates. They do so by means of a lexical rule that lifts objectlevel relations to kind-level relations, and incorporates the stereotypical usage of the kinds into the meaning of the resulting constructions. Let us summarize how this explains the properties of weak definites that were described in section 2 .

First, assuming that a weak definite is an ordinary definite, but referring to kinds, accounts for the presence of a definite article and the apparent lack of the uniqueness presupposition (1). Given that uniqueness applies at the level of kinds and given that instantiations of kinds can be atomic entities or sums, we expect weak definites to be felicitous even when more than one object entity in the context satisfies its descriptive content (13). Because of their kind-referring nature, it is also explained why weak definites can occur as the subject of a characterizing sentences (9). Similarly, it is expected that only some modifiers, namely, those that operate at the level of kinds allow weak interpretations (7).

Second, the way we represent the logical form of sentences with weak definites, illustrated in (20b), is consistent with weak definites not being able to establish discourse referents (12), because it does not involve existential quantification over individuals. With this kind of representations, sloppy identity in VP ellipsis (2) is due to the Th function being specific for each event (21). Narrow scope (3) is due to the same reason, because the event quantifier has narrow scope with respect to scope 
Weak definites and reference to kinds

bearing operators (22).

Third, the stereotypical usage of kinds represents the enriched meaning of the sentences with weak definites (10). Also, it accounts for the lexical restrictions these definites are subject to: only certain nouns (with the adequate number morphology) that support stable, specific and stereotypical patterns of use will trigger weak readings (5)-(6); only verbs and prepositions that are associated to the usage patterns of the kind that correspond to a weak definite will combine with that weak definite (4).

We believe that our proposal has some important virtues. On the one hand, it has taken a first step towards an analysis of a certain class of ill-understood weak definites, opening up new ways of looking at the role of the functional aspect of the meaning of nouns, the telic role along the lines of the qualia theory of Pustejovsky (1991), kind reference, and the use of the definite article. On the other hand, it explains the exceptional properties of weak definites with mechanisms well motivated in the literature such as reference to kinds, the realization relation, type shifts and the iota operator. Finally, it treats definite generics and weak definites as different faces of a same phenomenon, which is empirically and methodologically satisfactory.

Our approach also brings along a number of questions we would like to answer in future work. One of them has to do with the possible scope of our analysis. In principle we have aimed to account for the definites described by Carlson and Sussman only. However, we wonder whether we could extend the analysis to other phenomena of weak definiteness such as so-called possessive weak definites like the corner of a busy intersection (cf. Poesio 1994; Barker 2005), or definites that refer to body parts like the arm (Ojeda 1993; Levinson 2006). How fruitful would it be to treat them as kind referring expressions? How should we cope with the fact that they do not share some of the properties of weak definites, like the semantic enrichment and the lexical restrictions?

Another question involves the resemblance between weak definites and bare singular nominals like (be in) jail, prison, church and town, in several respects (i.e., narrowest scope, lexical restrictions, no modification, enriched meaning and no subject position). Are these expressions of the same type of weak definites? How then should we account for the presence/absence of the definite article? Are they rather manifestations of different but functionally related phenomena? Carlson (2006) and Stvan (1998) have argued that these bare singulars are cases of semantic incorporation, and similar kinds of special combining relations between verbs and objects have been proposed for other types of bare nouns in different languages (e.g. van Geenhoven 1998; Farkas \& de Swart 2003; Dayal 2003; Espinal \& McNally to appear). What is the connection (if any) between incorporation and pseudoincorporation and the kind of verb-object combination we have studied in this paper? 
Ana Aguilar-Guevara and Joost Zwarts

\section{References}

Abbott, Barbara. 2000. Definiteness and identification in English. In Enikö Németh (ed.), Pragmatics in 2000: Selected papers from the 7th international pragmatics conference, vol. 2, 1-15. Antwerp: International Pragmatics Association.

Barker, Chris. 2005. Possessive weak definites. In Ji-Yung Kim, Yury Lander \& Barbara Partee (eds.), Possessives and beyond: Semantics and syntax, 89-113. Amherst, MA: GLSA Publications.

Birner, Betty J. \& Gregory Ward. 1994. Uniqueness, familiarity, and the definite article in English. Berkeley Linguistics Society Annual Meeting (BLS) 20.93102.

Carlson, Gregory. 1977. Reference to kinds in English: University of Massachusets, Amherst dissertation.

Carlson, Gregory. 2006. The meaningful bounds of incorporation. In Svetlana Vogeleer \& Liliane Tasmowski (eds.), Non-definiteness and plurality, 35-50. John Benjamins Publishing Company.

Carlson, Gregory. \& Rachel Shirley Sussman. 2005. Seemingly indefinite definites. In Stephan Kepser \& Marga Reis (eds.), Linguistic evidence: Empirical, theoretical, and computational perspectives, 26-30. Berlin: Mouton de Gruyter.

Chierchia, Gennaro. 1998. Reference to kinds across language. Natural Language Semantics 6(4). 339-405. doi:10.1023/A:1008324218506.

Dayal, Veneeta. 2003. A semantics for pseudo-incorporation. Rutgers University. ms.

Dayal, Veneeta. 2004. Number marking and (in) definiteness in kind terms. Linguistics and Philosophy 27(4). 393-450. doi:10.1023/B:LING.0000024420.80324.67.

Epstein, Richard. 2002. The definite article, accessibility, and the construction of discourse referents. Cognitive Linguistics 12(4). 333-378. doi:10.1515/cog1.2002.007.

Espinal, Maria T. \& Louise. McNally. to appear. Bare nominals and incorporating verbs in Catalan and Spanish. Journal of Linguistics 47(2).

Farkas, Donka \& Henriette de Swart. 2003. The semantics of incorporation. CSLI Publications.

van Geenhoven, Veerle. 1998. Indefinite Descriptions and Semantic Incorporation: Semantic and Syntactic Aspects of Noun Incorporation in West Greenlandic. CSLI Publications.

Horn, Larry. 1984. A new taxonomy for pragmatic inference: Q-based and R-based implicatures. In Deborah Schiffrin (ed.), Meaning, form and use in context: Linguistic applications, 11-42. Georgetown University Press.

Kratzer, Angelika. 1996. Severing the external argument from its verb. In Johan 
Weak definites and reference to kinds

Rooryck \& Laurie Ann Zaring (eds.), Phrase structure and the lexicon, 109-137. Kluwer Academic Publishers.

Krifka, Manfred. 1995. Genericity: an introduction. In Gregory Carlson \& Francis J. Pelletier (eds.), The Generic Book, 1-124. The University of Chicago Press, Chicago.

Krifka, Manfred. 2003. Bare NPs: Kind-referring, indefinites, both, or neither? In Robert B. Young \& Yuping Zhou (eds.), Semantics and Linguistic Theory (SALT) XIII, 180-203. CLC Publications, Cornell.

Levinson, Dmitry. 2006. Definiteness of Body Part Terms in Spanish and Portuguese. Selected Proceedings of the 8th Hispanic Linguistics Symposium 172-182.

Levinson, Stephen C. 2000. Presumptive meanings: The theory of generalized conversational implicature. MIT Press.

Löbner, Sebastian. 1985. Definites. Journal of Semantics 4(4). 279. doi:10.1093/jos/4.4.279.

McNally, Louise \& Gemma Boleda. 2004. Relational adjectives as properties of kinds. Empirical Issues in Formal Syntax and Semantics 5. 179-196.

Nunberg, Geoffery, Ivan A. Sag \& Thomas Wasow. 1994. Idioms. Language 70(3). 491-538. doi:10.2307/416483.

Ojeda, Almerindo E. 1993. New evidence for a more general theory of singularity. ESCOL 93. 247-258.

Parsons, Terence. 1990. Events in the Semantics of English. Cambridge, MA: MIT Press.

Poesio, Massimo. 1994. Weak definites. Proceedings of the Fourth Conference on Semantics and Linguistic Theory, SALT 4.

Pustejovsky, James. 1991. The generative lexicon. Computational Linguistics 17(4). 409-441.

Russell, Bertrand. 1905. On denoting. Mind 14(56). 479-493. doi:10.1093/mind/XIV.4.479.

Scholten, Jolien \& Ana Aguilar-Guevara. 2010. Assessing the discourse referential properties of weak definite NPs. In Jacqueline van Kampen \& Rick Nouwen (eds.), Linguistics in the Netherlands, vol. 27, 115-128. John Benjamins. doi:10.1075/avt.27.10sch.

Schwarz, Florian. 2009. Two types of definites in natural language: University of Massachusetts, Amherst dissertation.

Strawson, Peter F. 1950. On referring. Mind 59(235). 320-344. doi:10.1093/mind/LIX.235.320.

Stvan, Laurel S. 1998. The semantics and pragmatics of bare singular noun phrases: Northwestern University dissertation. 
Ana Aguilar-Guevara and Joost Zwarts

Ana Aguilar-Guevara

Trans 10, 3512 JK

Utrecht, The Netherlands.

A.AguilarGuevara@uu.nl
Joost Zwarts

Trans 10, 3512 JK

Utrecht, The Netherlands.

J.Zwarts@uu.nl 